\title{
Casimir energy calculations within the formalism of the noncompact lattice QED
}

\author{
Oleg Pavlovsky ${ }^{\dagger}$ and Maxim Ulybyshev ${ }^{\ddagger}$ \\ Institute for Theoretical Problems of Microphysics, Moscow State University, Moscow, Russia \\ $\dagger$ †-mail: ovp@goa.bog.msu.ru $\ddagger$ E-mail: ulybyshev@goa.bog.msu.ru
}

\begin{abstract}
A new method based on the Monte-Carlo calculation on the lattice is proposed to study the Casimir effect in the noncompact lattice QED. We have studied the standard Casimir problem with two parallel plane surfaces (mirrors) and oblique boundary conditions on those as a test of our method. Physically, this boundary conditions may appear in the problem of modelling of the thin material films interaction and are generated by additional Chern-Simons boundary term. This approach for the boundary condition generation is very suitable for the lattice formulation of the Casimir problem due to gauge invariance.
\end{abstract}

Keywords: Lattice gauge theory, quantum electrodynamics, Casimir effect.

PACS numbers: 11.15.Ha, 12.20.Ds.

\section{Introduction and general motivation}

During the last few years Casimir effect has attracted much attention due to the great experimental and theoretical progress in studying of this phenomenon. This macroscopic quantum effect plays crucial role in nanophysics, micro-mechanics, quantum optics, condensed matter physics, material science, and also it is very important for different models of boundary states in hadron physics, heavy-ion collisions, and cosmology.

Nowadays, many theoretical methods for calculation of Casimir effect are proposed. Unfortunately, most of these methods are based on the fixed boundary conditions or external potentials. Such a simplification can lead to problems in gauge invariance, renormalizability and locality. Moreover, there are some problems with different thermal corrections to Casimir force: different methods of calculations of the Casimir effect predict different corrections. Another part of the problem is calculation of Casimir force for complicated forms of boundary surfaces. Typically, various approximate methods (like the proximity force approximation method [1], [2]) are used in the case of curved surfaces, but it is still unclear now whether those approximations are correct and for what tasks they can be applied. And finally, analytic methods for Casimir effect calculation are very complicated and strongly dependent on the shape of surfaces. Practically, Casimir effect was studied analytically only for cases of plane, spherical and cylindrical surface forms. More complicated tasks have not been studied very well by this moment but such cases typically appear in experiments.

Based on arguments discussed above, it seems a very important task now to create a general method for calculation of Casimir effects which would work well for different shapes of boundary surfaces, at non-zero temperature and density and under other external factors. It means that such a method should be formulated very generally for working in different coupling regimes and different external conditions. And we think that direct lattice calculations in quantum field theory can meet all these requirements. 
In our paper we consider the simplest Casimir problem with two parallel planes as a test of our method. The existence of the analytical answer for this problem is the additional motivation for such a choice of bound surfaces. This analytical answer assists us in fitting procedure for our numerical results.

A crucial obstacle on the way of the realization of any Casimir problem on the lattice is the following. If the Casimir energy is a reaction of the vacuum on the presence of the boundary, what is "the boundary" in terms of lattice formalism? In other words, what is an observable quantity corresponding to such boundary? In fact, the answer is non-trivial and we devote the first part of the paper to this question. In the second part, we discuss the lattice algorithms and numerical results. And the last part is a conclusion.

\section{Chern-Simons boundary conditions and Casimir effect}

Casimir effect is a reaction of the vacuum on boundary condition. The spectrum of vacuum fluctuations depends on the boundary conditions. Changing of the boundary conditions leads to changing of the spectrum of vacuum fluctuations and so to generating of the corresponding Casimir force on the boundary. In the standard quantum field theory formalism, such changing of spectrum of vacuum fluctuations can be described, for example, by means of Green function method [1]. This approach is a very powerful tool for studying many essential Casimir tasks [1. Unfortunately, the application of this analytical method to the case of more complicated shape of the boundary surfaces is not so easy due to calculation difficulties. Our aim is the creation of the numerical method for the Casimir effect calculation directly from the quantum field theory action. The lattice formalism looks very attractive for this role but manifestly we can not base in our approach on the separation of vacuum modes corresponding with boundary from the full spectrum of vacuum fluctuation. In lattice formalism we work in Euclidian space and deal with full spectrum of vacuum fluctuations and can not easily snatch out vacuum fluctuations corresponding to some boundary conditions. We need some very delicate approach for separation of vacuum fluctuation modes that preserve gauge invariance of our lattice formalism. Fortunately, such an approach to Casimir problem was proposed recently [ ․, 6].

This approach is based on a very elegant idea coming from some unique properties of the Chern-Simons action in three dimensions [5, 6]. Let us consider electro-magnetic fields in $3+1$ dimensions with the Maxwell action and additional Chern-Simons action given on 3-dimensional integral on the boundary surface $S$ :

$$
S=-\frac{1}{4} \int d^{4} x F_{\mu \nu} F^{\mu \nu}-\frac{\lambda}{2} \oint d^{3} s \varepsilon^{\sigma \mu \nu \rho} n_{\sigma} A_{\mu}(x) F_{\nu \rho}(x),
$$

where $\varepsilon^{\sigma \mu \nu \rho}$ is the Levi-Civita tensor and $n_{\sigma}$ is the normal vector to the boundary surface $S, \lambda$ is a real parameter.

Let us consider now the simplest form of boundary surface $S$, namely two parallel infinite planes placed at the distance $R$ from each other. The Chern-Simons formulation of this canonical Casimir problem was studied analytically in series of works [10, 11]. We will use this analytical answer for the fitting of our numerical data.

In the case of plane form of the boundary surface $S$ the Chern-Simons action in (1) has the following form:

$$
S_{C S}=\frac{\lambda}{2} \int\left(\delta\left(x_{3}\right)-\delta\left(x_{3}-R\right)\right) \varepsilon^{3 \mu \nu \rho} A_{\mu}(x) F_{\nu \rho}(x) d^{4} x .
$$

where, in our formulation of this Casimir problem, normal vectors to the planes are turned in the opposite directions. This choice of the normal vector orientation corresponds to our renormalization procedure based on the connection between open and closed Casimir problems. 


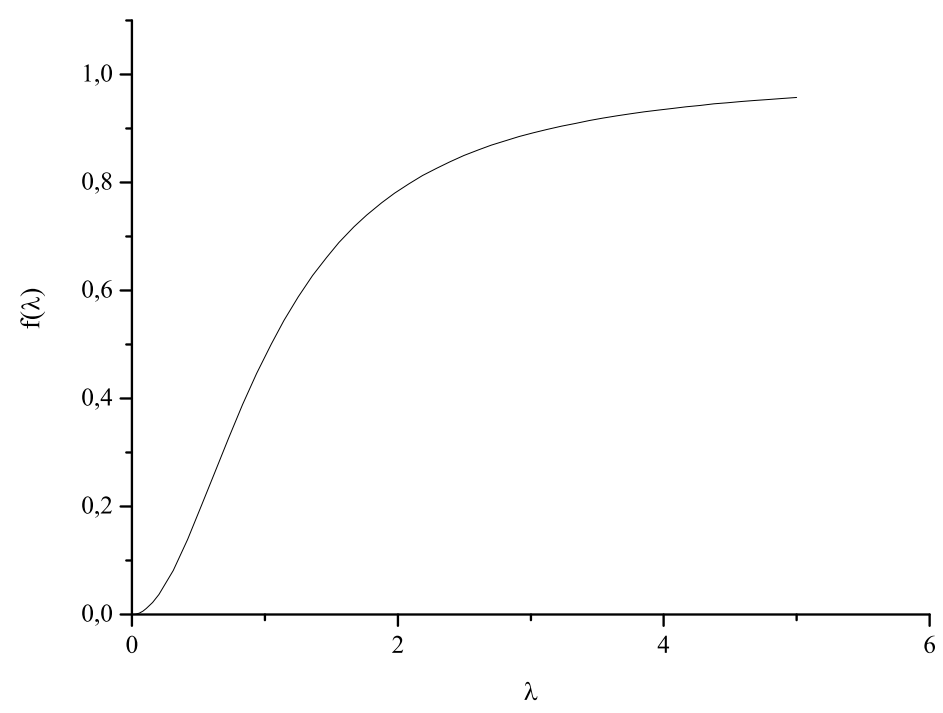

Figure 1: $f(\lambda)$.

If the parameter $\lambda$ is small, electro-magnetic fields obviously don't feel any boundary and are free. What happens if the parameter $\lambda$ becomes large and tends to infinity and fields dynamics on the boundary surface $S$ is determined by Chern-Simons action? Let us consider the equation of motion obtained from the action (1):

$$
\square A^{\mu}+\lambda\left(\delta\left(x_{3}\right)-\delta\left(x_{3}-R\right)\right) \varepsilon^{3 \sigma \nu \rho} A_{\sigma} \partial_{\nu} A_{\rho}=0 .
$$

At $\lambda \rightarrow \infty$, it is easy to obtain from (3) a corresponding boundary conditions on the surface $S$ :

$$
\left.E_{\|}\right|_{S}=0,\left.\quad H_{n}\right|_{S}=0,
$$

where $H_{n}$ and $E_{\|}$are normal and longitudinal components of magnetic and electric fields correspondingly. These conditions mean the nulling of the energy flux of the electromagnetic field through the surface.

This phenomenon corresponds to the well-known property of the Chern-Simons theory in 3dimension, namely to topological vortex (strings) generation [7, 8]. As it was shown by Witten [9], Chern-Simons theory, which is a quantum field theory in three dimensions, is exactly solvable by using nonperturbative methods and the topological vortices play a leading role in this process. It was shown that the partition function of this theory depends on the topology of the surface $S$ and gauge group only, and tends to the zero if $\lambda$ tends to infinity. There are no propagation modes in this case and if the boundary surface $S$ is closed, the gauge fields inside and outside this surface become separated from each other by the topological vortex fields on the surface. In the case of the finite value of $\lambda$, we have a non-trivial interaction between topological vortices and electro-magnetic waves [8]. For our practical purposes it is enough to know that the parameter $\lambda$ plays the role of the effective regulator of the penetrability of our boundary surface for electro-magnetic wave.

The analytical answer of Casimir energy per unit area for two planes is given by Green function method is the following [10, 11]:

$$
E_{c a s}=-\frac{\pi^{2}}{720 R^{3}} f(\lambda),
$$


where function $f(\lambda): \lim _{\lambda \rightarrow \infty} f(\lambda)=1$ (Fig. 1) can be written as:

$$
f(\lambda)=\frac{90}{\pi^{4}} \operatorname{Li}_{4}\left(\frac{\lambda^{2}}{\lambda^{2}+1}\right)
$$

here the polylogarithm function $\operatorname{Li}_{4}(x)$ is defined as

$$
\operatorname{Li}_{4}(x)=\sum_{k=1}^{\infty} \frac{x^{k}}{k^{4}}=-\frac{1}{2} \int_{0}^{\infty} k^{2} \ln \left(1-x e^{-k}\right) d k .
$$

In our paper we will study the Casimir energy per unit area behavior at small $\lambda$, which is the following:

$$
E_{c a s}=-\frac{\lambda^{2}}{8 \pi^{2} R^{3}}+O\left(\lambda^{4}\right)
$$

\section{Wilson "bag" and numerical lattice simulation of Maxwell- Chern-Simons theory}

\subsection{General statements}

In our paper we use the four-dimensional hyper-cubical lattice in Euclidian space-time and the simplest form of the action for the noncompact lattice QED:

$$
S=\frac{\beta}{2} \sum_{x} \sum_{\mu<\nu} \theta_{p, \mu \nu}^{2}(x),
$$

where the link and plaquette variables are defined as:

$$
\begin{gathered}
\theta_{l, \mu}(x)=\text { e } a A_{\mu}, \\
\theta_{p, \mu \nu}(x)=\triangle_{\mu} \theta_{l, \nu}(x)-\triangle_{\nu} \theta_{l, \mu}(x), \\
\triangle_{\mu} \theta_{l, \nu}(x)=\theta_{l, \nu}(x+\hat{\mu})-\theta_{l, \nu}(x) .
\end{gathered}
$$

Here $a$ is a lattice step and the parameter $\beta=1 / e^{2}$. Physical quantities are calculated in the lattice formalism by means of field configuration averaging, where the field configurations (the set of all link variables) are generated with the statistical weight $e^{-S}$.

We have clarified in previous sections that additional Chern-Simons action describes Casimir effect. In order to find a lattice description of Casimir interaction between boundary surfaces let us consider Wilson loop, which describes the interaction of charged particles. Wilson loop can be written in QED as

$$
W_{C}=e^{i g \oint_{C} A_{\mu} d x_{\mu}}=e^{i \int J_{\mu} A_{\mu} d x^{4}} .
$$

The exponent in (77) is the additional term to the action. This term describes the interaction

of the field $A_{\mu}$ with the current $J_{\mu}(x)=g \oint_{C} \delta(x-\xi) d \xi_{\mu}$ of charged particle. Configuration averaging of Wilson loop $\langle W(R, T)\rangle$ (where $\mathrm{R}$ and $\mathrm{T}$ are dimensions of the loop) converges in Euclidian time in the limit $T \rightarrow \infty$ to:

$$
\langle W(R, T)\rangle \rightarrow C e^{-V(R) T},
$$

where $V(R)$ is the energy of interaction between charged particles. The same method can be used for calculation of Casimir energy by means of Chern-Simons action. 


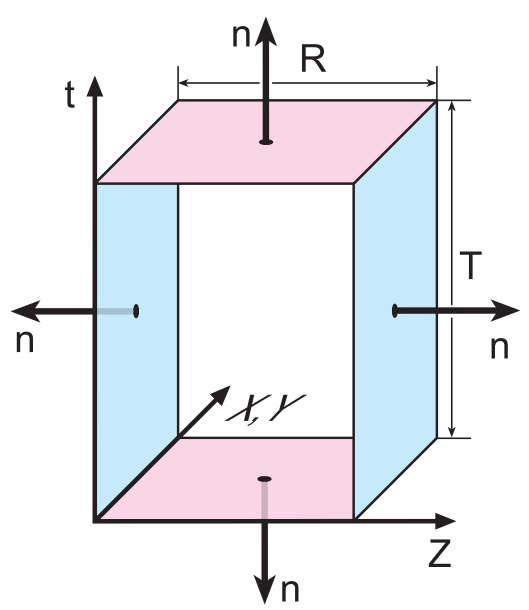

Figure 2: Wilson bag for two plane surfaces.

Analogously to the description of charged particles interaction by 1D integral along Wilson loop, we will describe the Casimir interaction of surfaces by corresponding 3D integral. The first problem is that for stationary objects the action (2) is an integral from $t=-\infty$ to $t=\infty$, so by analogy to Wilson loop, we should enclose the surface of the integration in t-direction. The integration surface for two planes is shown in Fig. 2. This closing procedure can be performed both for plane surfaces and for any curved surface in 3-dimensional space. As the result of this procedure, so-called Wilson bag [3, 4] can be obtained. It can be written as

$$
e^{i \lambda \oint_{\Sigma} \varepsilon_{\mu \nu \rho \sigma} A_{\nu} F_{\rho \sigma} d S_{\mu}}
$$

where $\Sigma$ is closed 3-dimensional surface in 4-dimensional space-time. The final conclusion is that Wilson bag is (by analogy to Wilson loop) observable quantity which gives us Casimir energy of the objects, defined by the surface of integration. For two planes we will calculate the following object:

$$
W_{B a g}(R, T)=e^{i \lambda S(R, T)}
$$

where

$$
\begin{gathered}
S(R, T)=\int_{0}^{T} d t \iiint d x d y d z(\delta(z-R)-\delta(z)) \varepsilon_{3 \nu \rho \sigma} A_{\nu} F_{\rho \sigma}+ \\
+\int_{0}^{R} d z \iiint d x d y d t(\delta(t-T)-\delta(t)) \varepsilon_{4 \nu \rho \sigma} A_{\nu} F_{\rho \sigma} .
\end{gathered}
$$

And in the limit $T \rightarrow \infty$ :

$$
\left\langle W_{\text {Bag }}(R, T)\right\rangle \rightarrow C e^{-E_{c a s}(R) T} .
$$

The second problem is to rewrite the Wilson bag in terms of lattice objects (links and plaquettes). The product

$$
A_{\nu} F_{\rho \sigma}
$$

can be exactly constructed only in non-compact QED, because just in the non-compact QED the link variable $\theta_{l, \nu}$ is a lattice analogue of $A_{\nu}$ and the plaquette variable $\theta_{p, \mu \nu}$ is a lattice analogue of $F_{\mu \nu}$.

There are two requirements for lattice representation of the Wilson bag: 


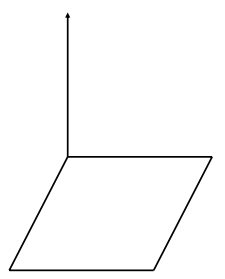

Figure 3: "Corner": the basic element for the building of CS lattice action.

1) The whole integral for Wilson bag should be the gauge invariant quantity.

2) "Locality". It means that in lattice representation of the product $A_{\nu} F_{\rho \sigma}, A_{\nu}$ and $F_{\rho \sigma}$ should be given in the same point $x$. This requirement is non-trivial, because $\theta_{p, \rho \sigma}$ gives the value of $F_{\rho \sigma}$ in the center of the plaquette, but $\theta_{\nu}$ gives the value of $A_{\nu}$ in the center of the link. These are different points.

\subsection{Gauge invariance}

A basic element for building of the CS lattice action will be a "corner" (Fig. 3). It is a product of the link variable and one of the neighboring plaquette variables. This product is a lattice analogue of the product $A_{\nu} F_{\rho \sigma}$.

First we will show how to construct the gauge invariant quantity from these basic elements. Let's consider one CS plane closed over the lattice period in all directions $\left(V_{3}\right)$. The "naive" discretization of this quantity in Euclidean space-time is follows:

$$
S_{C S}=\sum_{x \in V_{3}} \varepsilon_{\mu \nu \rho \sigma} n_{\mu} \theta_{l, \nu}(x) \theta_{p, \rho \sigma}\left(x+\widehat{d x}_{\rho \sigma}\right),
$$

where the vector $\widehat{d x}_{\rho \sigma}$ appoints to the plaquette that attached to the link $\theta_{l, \nu}(x)$ :

$$
\begin{gathered}
\widehat{d x}_{\rho \sigma}=c_{\rho \sigma} \hat{\nu}-a_{\rho \sigma} \hat{\rho}-b_{\rho \sigma} \hat{\sigma}, \\
a_{\rho \sigma}, b_{\rho \sigma}, c_{\rho \sigma}=0,1 .
\end{gathered}
$$

There are 8 variants how to join a link with one of the neighboring plaquettes, two of them one can find in Fig. 4.

Now let us consider gauge transformation of the "naive" lattice CS action (10). The gauge transformation of the link variable is follows:

$$
\theta_{l, \nu} \rightarrow \theta_{l, \nu}+\alpha(x+\hat{\nu})-\alpha(x)
$$

and the action (10) transforms into the following expression due to the closing over the lattice period:

$S_{C S} \rightarrow \sum_{x \in V_{3}} \varepsilon_{\mu \nu \rho \sigma} n_{\mu} \theta_{l, \nu}(x) \theta_{p, \rho \sigma}\left(x+\widehat{d x}_{\rho \sigma}\right)+\sum_{x \in V_{3}} \varepsilon_{\mu \nu \rho \sigma} n_{\mu} \alpha(x)\left(\theta_{p, \rho \sigma}\left(x+\widehat{d x}_{\rho \sigma}-\hat{\nu}\right)-\theta_{p, \rho \sigma}\left(x+\widehat{d x}_{\rho \sigma}\right)\right)$.

The second term in (11) appears due to the gauge transformation. To formulate the lattice CS action in gauge invariant form, this second term must be equal to zero. It is possible to show that this term is equal to zero if plaquettes in it are the sides of one $3 \mathrm{~d}$ cube for all lattice sites $x$ and normal vectors $n_{\mu}$. We should choose a variant to join a link and a plaquette which has the property given above. One example of such a join is shown in Fig. 5. 


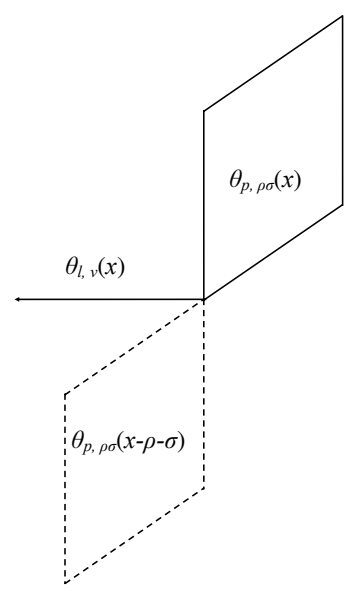

Figure 4: Two types of "corners".

This combination of "corners" is organized as follows: for every 3d cube the "corners" of different orientations that lie inside it (it means that "corners" are built from edges of this cube) have theirs link free ends being at one lattice site. This structure can be obtained for example by the following choice of the vector $\widehat{d x}_{\rho \sigma}$ :

$$
\widehat{d x}_{\rho \sigma}=-\hat{\rho}-\hat{\sigma}
$$

This choice provides the nulling of the expression:

$$
\varepsilon_{\mu \nu \rho \sigma}\left(\theta_{p, \rho \sigma}\left(x+\widehat{d x}_{\rho \sigma}-\hat{\nu}\right)-\theta_{p, \rho \sigma}\left(x+\widehat{d x}_{\rho \sigma}\right)\right)=0, \forall x, \mu .
$$

Thus the Chern-Simons action obtained from such a system of "corners" is the gauge invariant.

One can perform the similar consideration for any closed 3-dimensional surface. An example of such a closing for the "corner" with some orientation one can find in Fig. 6. Every "corner" should be a part of such a chain and every chain should be closed.

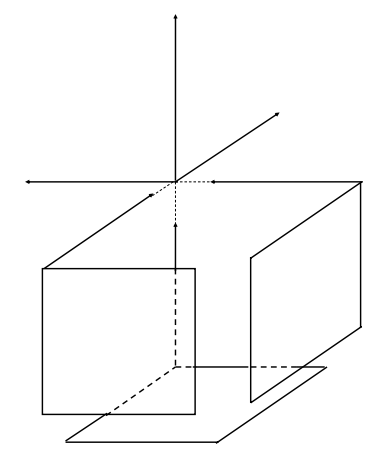

Figure 5: Combination of "corners" with different orientations that leads to the gauge invariant lattice Chern-Simons action. 


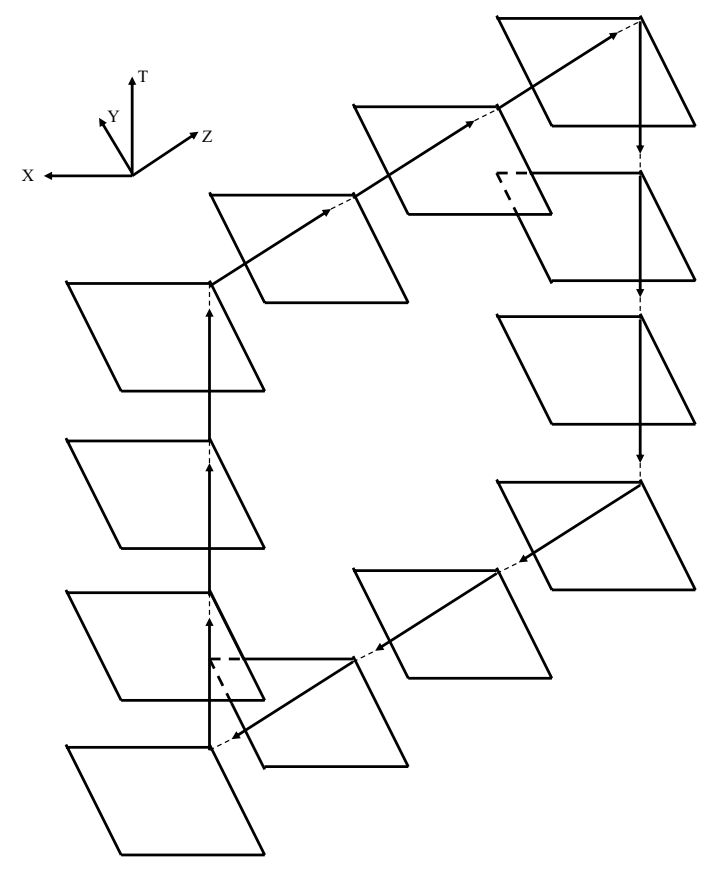

Figure 6: Closing for the "corner" of one orientation.

\subsection{Locality}

The next point of our consideration is the "locality". As it is well known, the plaquette variable $\theta_{p, \rho \sigma}$ gives us the value of $F_{\rho \sigma}$ in the center point of the plaquette, while the link variable $\theta_{l, \mu}$ gives us the $A_{\mu}$ in the middle point of the link. On the other hand, the averages

$$
1 / 4\left(\theta_{l, \nu}(x)+\theta_{l, \nu}(x+\hat{\rho})+\theta_{l, \nu}(x+\hat{\sigma})+\theta_{l, \nu}(x+\hat{\rho}+\hat{\sigma})\right)
$$

and

$$
1 / 2\left(\theta_{p, \rho \sigma}(x)+\theta_{p, \rho \sigma}(x+\hat{\nu})\right)
$$

give us the values of $A_{\nu}$ and $F_{\rho \sigma}$ in the center of the 3-dimensional cube determined by the 3 vectors $\hat{\rho}, \hat{\nu}$ and $\hat{\sigma}$ based on one site $x$.

It's obvious that the product of these two averages is the same as the sum of all 8 variants of "corners", in other words we should use all possible kinds to join of a link and a plaquette inside one $3 \mathrm{~d}$ cube. These 8 variants are shown on Fig. 7 for one possible orientation of a "corner". For another two possible orientations one has a similar set of variants.

Let us consider now all 3 orientation of "corners". For each of them there is the set of 8 variants of possible join a link with a plaquette. On other hand one can say that we have 8 systems of "corners" (3" corners" in one system). Each of these systems is similar to the system of "corners" shown in the Fig. 5 (links' free ends of all 3 "corners" are collected in one vertex of a cube) and forms the gauge invariant quantity after summation over the closed 3-dimensional surface.

Finally, our lattice representation of CS action is the sum over all possible variants of the 

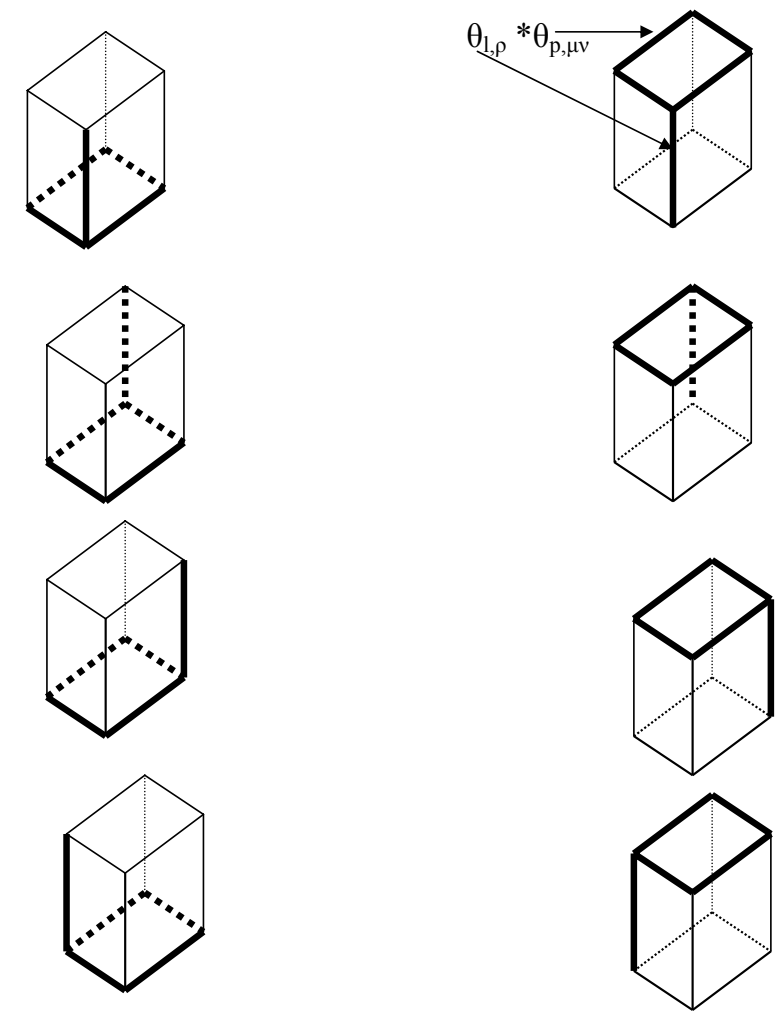

Figure 7: The structure of integral for "Wilson bag". To obtain the gauge invariant quantity we sum inside its 3-dimensional volume over all the products of plaquette and neighboring link. All these variants for one possible orientation of link and plaquette inside one 3-dimensional cube are shown here.

"corners" for three different orientations inside the close 3-dimensional surface:

$S_{C S}=\frac{1}{8} \beta \sum_{x \in V_{3}} \varepsilon_{\mu \nu \rho \sigma} n_{\mu}(x)\left(\theta_{l, \nu}(x)+\theta_{l, \nu}(x+\hat{\rho})+\theta_{l, \nu}(x+\hat{\sigma})+\theta_{l, \nu}(x+\hat{\rho}+\hat{\sigma})\right)\left(\theta_{p, \rho \sigma}(x)+\theta_{p, \rho \sigma}(x+\hat{\nu})\right)$.

The factor $1 / 8$ in (12) comes from the averaging over the 8 variants of the link and plaquette joining. The expression $\theta_{l, \nu} \theta_{p, \rho \sigma}$ gives us $a^{3} e^{2} A_{\nu} F_{\rho \sigma}$ in continuum limit and by means of the factor $\beta=1 / e^{2}$ one can eliminate $e^{2}$ from the action.

\subsection{Deformed lattice}

To improve the accuracy we have used the lattice deformed in one direction. This formalism is quite similar to one that was used in article [14] but instead of t-direction we deform one of spatial directions (for definiteness z-direction). Briefly this formalism can be described as follows:

All link variables are connected with the field $A_{\mu}$ by the standard way:

$$
\theta_{l, \mu}=\text { e a } A_{\mu}, \mu=1,2,4
$$


except links in z-direction:

$$
\theta_{l, 3}=\text { oe } a A_{3}
$$

Here $a$ is the lattice step in all directions except $\mathrm{z}$ and $\alpha a$ is the lattice step in z-direction. The action of this theory can be written in the following way:

$$
S=\frac{\beta}{2}\left\{\alpha \sum_{x} \sum_{\mu<\nu ; \mu, \nu \neq 3} \theta_{p, \mu \nu}^{2}(x)+\frac{1}{\alpha} \sum_{x} \sum_{\nu} \theta_{p, 3 \nu}^{2}(x)\right\} .
$$

The additional Chern-Simons action is not changed on the a such deformed lattice. If $n_{\mu}$ (normal $4 \mathrm{~d}$ vector to the $3 \mathrm{~d}$ surface of "Wilson bag") is oriented along the z-direction, deformed links don't participate in "corners" and nothing changes. If $n_{\mu}$ is oriented along one of non-deformed axes, one deformed link always participates in link or plaquette variable of every "corner". For example:

$$
\begin{aligned}
& \theta_{l, 3} \theta_{p, 12}=e^{2} \alpha a^{3} A_{3} F_{12}, \\
& \theta_{l, 2} \theta_{p, 13}=e^{2} \alpha a^{3} A_{2} F_{13} .
\end{aligned}
$$

But we don't need to divide by $\alpha$, because this automatic presence of $\alpha$ in this expression simply corresponds to change of elementary lattice 3-dimensional volume in this part of "Wilson bag". Before deformation it was lattice $3 \mathrm{~d}$ cube with volume $a^{3}$. After deformation it's rectangular parallelepiped. Its edges have lengths $a, a$ and $\alpha a$, so its volume is $\alpha a^{3}$.

\section{Results of numerical calculations}

In our numerical simulations we calculate the Wilson bag quantity (8) with the lattice representation of $S_{C S}$ given by the formula (12). The Wilson bag surface is closed over the lattice period in x- and y-directions. $E_{\text {cas }}$ is extracted from the limit (9).

First we study the dependence of $E_{\text {cas }}$ on $R$ (distance between plates). In the Fig. 8 one can see $E_{\text {cas }}(R)$ dependence calculated on the lattice with dimensions 12 in $\mathrm{x}$ and $\mathrm{y}$-directions, 24 in z-direction (in order to decrease correlations over the lattice period in z-direction) and 72 in t-direction (in order to eliminate temperature effects). We did't use deformation in this calculation. Parameter $\lambda$ is equal to 0.02 here.

For this calculation we use $\beta=4$, but indeed in our Casimir calculations nothing depends on $\beta$. Casimir effect without radiational corrections doesn't depend on the electron charge in the continuous theory. On the lattice the independence appears due to the following reasons: 1 ) there is no phase transition in the non-compact lattice QED and $\beta$ here only plays the role of the scale parameter for a numerical value of link variables; 2 ) we eliminate this dependence from $S_{C S}$ due to the multiplication by $\beta$ in the final expression (12). The absence of the dependence was also tested numerically. So $\beta$ can be chosen in rather a large interval according to calculational convenience.

It's well known in the continuous theory [1] that $E_{\text {cas }}$ should depend on $R$ as $C / R^{3}$. So we'll try to fit the point sequence obtained from the numerical simulation by the function

$$
E_{\text {cas }}=P_{1}+\frac{P_{2}}{R^{3}}
$$

Our aim is, of course, the coefficient $P_{2} . P_{1}$ is an analog of something like self energy of charged particles. Such self energy can also be obtained in Wilson loop calculation. The insertion in the Figure 8 presents the full dependence from $R=1$ till $R=6$. The main graphic is the increased segment from $R=2$ till $R=6$. The red line is the fitting that was done for points $R=2 \ldots 6$ 
on the model function (13). One can see that numerical points at lattice distances $R=2 \ldots 6$ lie on the fitting rather well. The point $R=1$ differs from the normal $C / R^{3}$ dependence due to lattice artefacts at small distances.

The values of coefficients $P_{1}$ and $P_{2}$ are given in the Figure 8 . Now let us consider the physical meaning of the coefficient $P_{2}$. A full Casimir energy of the interaction between two planes have been obtained in these calculations. This energy is expressed in units $a^{-1}$, where $a$ is the lattice step. If we take into account that the area of the plane is equal to $(a N)^{2}$, where $N=12$ is the size of the lattice in $\mathrm{x}$ and $\mathrm{y}$-directions, we can write the physical (dimensional) value of the Casimir energy density:

$$
E_{\text {cas.phys }}=\frac{1}{a} \frac{P_{2}}{R^{3}} \frac{1}{(a N)^{2}}=\frac{P_{2} N^{-2}}{(R a)^{3}} .
$$

After comparison between this formula and (5) we can conclude that $P_{2} N^{-2}$ should be equal to coefficient at $R^{-3}$ in (5) or in (6) ( $\lambda$ is small in our calculations by reasons discussed later). And these values are rather close. $P_{2} N^{-2}=-4.44 \times 10^{-6} \pm 7 \times 10^{-8}$ and the theoretical value of this quantity for $\lambda=0.02$ is $-5.066 \times 10^{-6}$.

In order to improve the accuracy of calculation and obtain more points for the fitting procedure, we consider the lattice deformed in z-direction. As the planes are separated in zdirection, we'll be able to study $E_{c a s}(R)$ dependence more carefully. In the Fig.9 one can see $E_{\text {cas }}(R)$ obtained on the lattice with following parameters:

- 12 normal steps (the length of each step is $a$ ) in $\mathrm{x}$ and y direction.

- 72 normal steps in t-direction.

- 120 small steps (the length of each one is $\alpha a, \alpha=0.2$ ) in z-direction.

So the whole volume and dimensions of this lattice are the same that the previous one. The only difference is smaller step in z-direction.

The fitting procedure was realized again starting from distance $R=2 a=10(\alpha a)$ (2 nondeformed steps is equal to 10 deformed steps). As in the previous case, numerical points are very close to the fitting function. There is one peculiarity which we want to mention. The energy is expressed in the units $a^{-1}$, because we didn't change the step in t-direction, but $R$ is expressed now in the units $\alpha a$. So if the $E_{\text {cas }}(R)$ dependence is really close to the $C / R^{3}$ function, $P_{2}$ coefficient in fitting function (13) on the deformed lattice should be divided by $\alpha^{3}$ to obtain the $P_{2}$ coefficient on the non-deformed lattice. And these numerical values of coefficients really satisfy this condition.

The next step is the analysis of $P_{2}$ dependencies on the parameter $\lambda$ and on the size of the lattice. The first one is presented in the Fig. 10. In accordance with (6) , the calculated coefficient at $R^{-3}$ is proportional to $\lambda^{2}$ for small $\lambda$. Unfortunately, numerical errors increase rapidly for large $\lambda$ and in present calculations we are limited to small values of $\lambda$.

The dependence of $P_{2}$ on the lattice size is shown in Fig. 11. $P_{2}$ is proportional to $N^{2}$, where $N$ is the size of the lattice in $\mathrm{x}$ and y-direction. So the calculated Casimir energy of two planes is really proportional to theirs area.

The last point is the consideration of the continuous limit of our calculations. As we mentioned above nothing depends on the parameter $\beta$, due to Casimir energy independence of electron charge. So the only considerable stage in continuous limit is to go to the "large lattice" limit where size of the lattice $N \rightarrow \infty$. There is one feature in this continuous limit procedure and we think that this moment should be emphasized. The lattice step $a$ disappears from our considerations because the final result of our calculation is the dimensionless coefficient at $R^{-3}$ 
in expression for Casimir energy per unit area. So we don't need to define the physical volume of the lattice and the value of $a$ is not important for us. Because of arguments discussed above the continuous limit for $P_{2}$ coefficient is not sensitive to the limit $a \rightarrow 0$ and we have only the limit $N \rightarrow \infty$. $E_{\text {cas }}(R)$ dependence in Fig. 8. can be an illustration of this continuous limit: the correct dependence $E_{\text {cas }} \sim R^{-3}$ reveals only at lattice distances $R \geq 2$.

\section{Conclusions}

In this paper we have proposed the numerical method for the Casimir energy calculation based on the lattice simulations of QED. We have combined three ideas:

- The generation of the boundary conditions by means of the additional Chern-Simons boundary action and new lattice discretization of this additional action.

- The lattice "Wilson bag" concept (the lattice presentation of the closed 3-dimensions surface in Euclidian 4-dimensions space).

- The improvement of the numerical results by using deformed lattice in space direction.

The combination of the first two ideas is in fact a lattice definition of the respective quantum observable for the Casimir energy and for the Casimir interaction between surfaces and last one is a very important numerical tool to archive the necessary accuracy.

We have tested our method in the simplest case of the Casimir interaction between two plane surfaces and have achieved results close to the analytical ones for this problem.

\section{Acknowledgments}

We thank Prof. V.V. Nesterenko, Dr. I.G. Pirozhenko, Dr. M.A. Trusov and Dr. V.N. Marachevsky for interesting discussions about the Casimir problem and Andrew Zayakin for essential remarks about vortex dynamics of the Chern-Simons theory. The resources of MSU supercomputer center were used in our calculations. The work is partially supported by the Russian Federation President's Grant 195-2008-2.

\section{References}

[1] M. Bordag, U. Mohideen and V. M. Mostepanenko, Phys. Rept. 353 (2001) 1.

[2] B.V. Deryagin, I.I. Abrikosova and E.M. Lifshitz, Quart. Rev. Chem. Soc. 10 (1958) 295; Uspekhi Fiz. Nauk 64 (1958) 493.

[3] N. Seiberg, Phys. Lett. 148 (1984) 456.

[4] M. Luscher, Phys. Lett. B 78 (1978) 465.

[5] M. Bordag and D. V. Vassilevich, Phys. Lett. A 268 (2000) 75.

[6] E. Elizalde and D. V. Vassilevich, Class. Quant. Grav. 16 (1999) 813.

[7] M. Marino, Rev. Mod. Phys. 77 (2005) 675.

[8] P. A. Horvathy, arXiv:0704.3220 [hep-th]. 
[9] E. Witten, Commun. Math. Phys. 121 (1989) 351.

[10] V. N. Markov and Yu. M. Pis'mak, J. Phys. A 39 (2006) 6525.

[11] V. N. Markov and Yu. M. Pis'mak, arXiv:hep-th/0505218.

[12] A. Hosaka and H. Toki, Phys. Rept. 277 (1996) 65.

[13] I. Y. Malakhov and K. A. Sveshnikov, Theor. Math. Phys. 132 (2002) 1201.

[14] G. Burgers and F. Karsch Nucl. Phys. B 304 (1988) 587. 


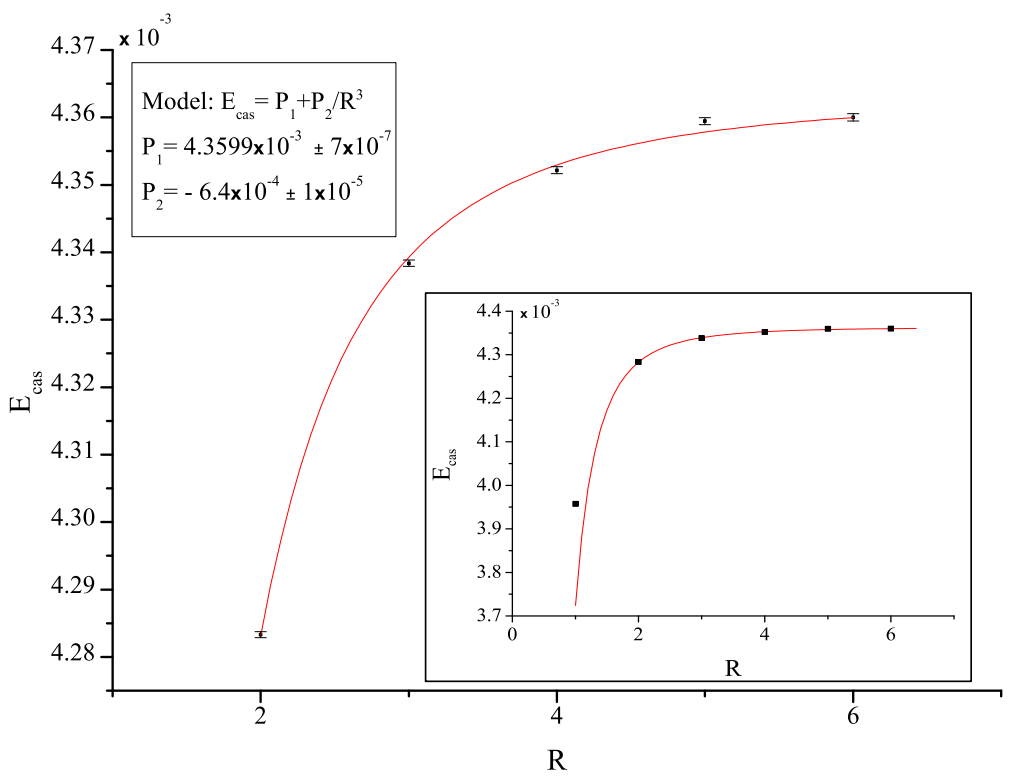

Figure 8: $E_{\text {cas }}(R)$ at $\beta=4.0$ and $\lambda=0.02$.



Figure 9: $E_{\text {cas }}(R)$ dependence at $\beta=4.0$ and $\lambda=0.02$ on deformed lattice. Step in z-direction is $\alpha a$, in other directions it is $a, \alpha=0.2$. 


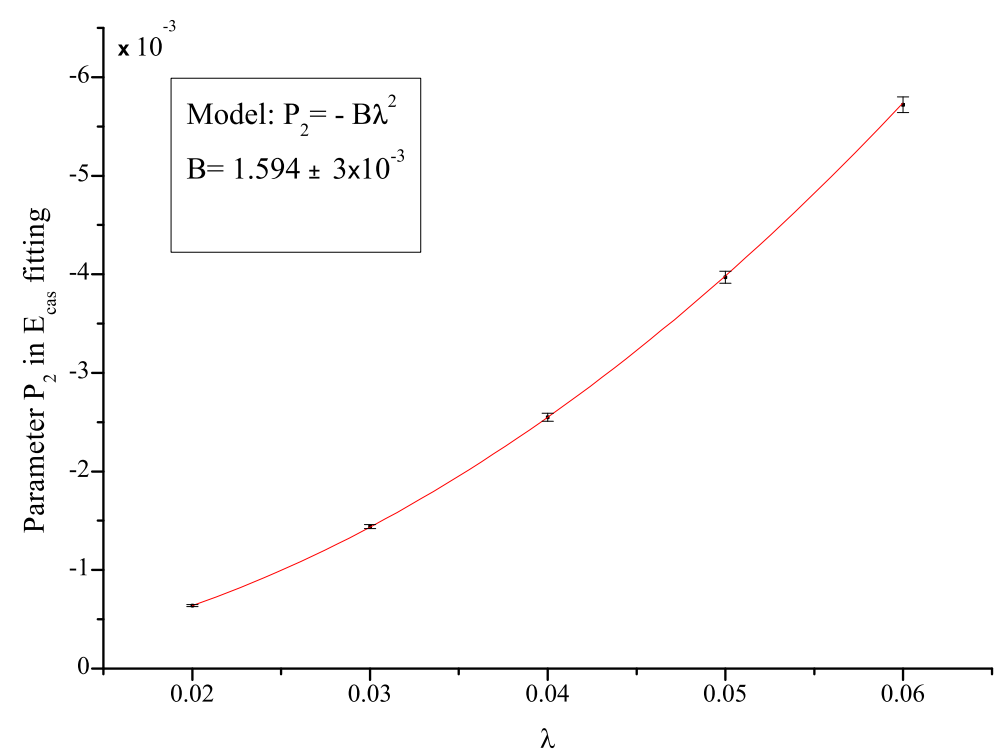

Figure 10: $P_{2}(\lambda)$ dependence. Lattice is the same that was used in Fig. 1.

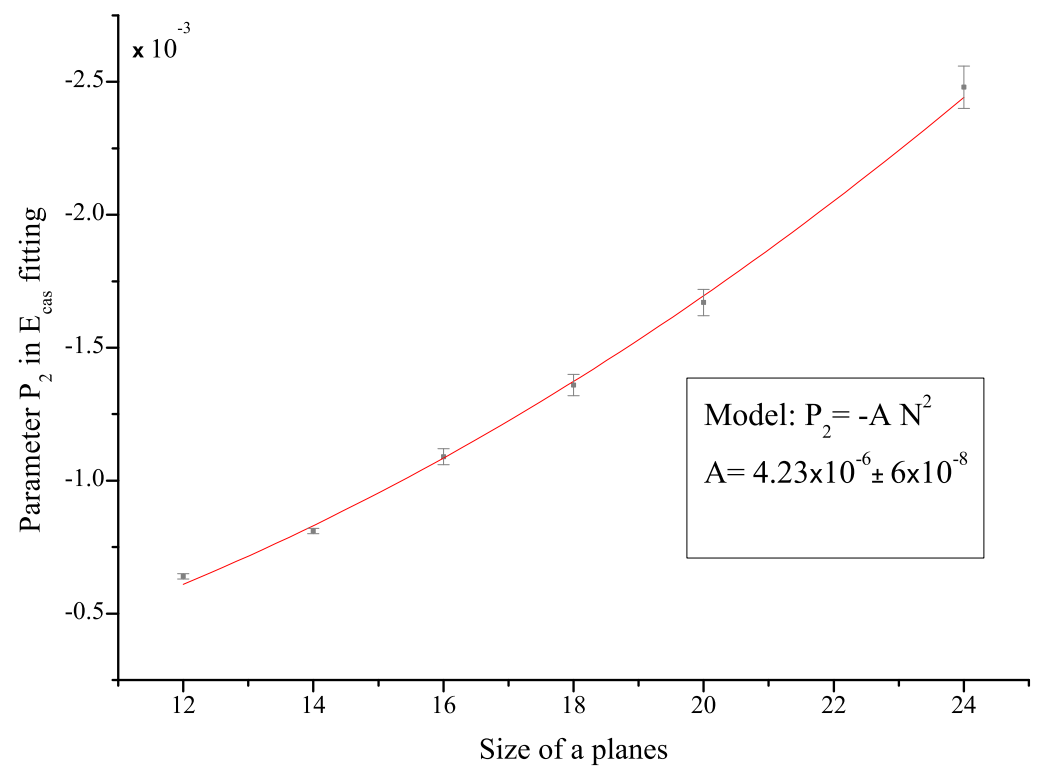

Figure 11: $P_{2}(N)$ dependence. $N$ is the size of the lattice in $\mathrm{x}$ and $\mathrm{y}$-directions. Lattice without deformations, $\beta=4.0$ and $\lambda=0.02$. 